\title{
Semantic transparency effects in German compounds: A large dataset and multiple-task investigation
}

\author{
Fritz Günther ${ }^{1}$. Marco Marelli ${ }^{1,2}$ • Jens Bölte ${ }^{3}$ \\ Published online: 12 February 2020 \\ (C) The Psychonomic Society, Inc. 2020
}

\begin{abstract}
In the present study, we provide a comprehensive analysis and a multi-dimensional dataset of semantic transparency measures for 1810 German compound words. Compound words are considered semantically transparent when the contribution of the constituents' meaning to the compound meaning is clear (as in airport), but the degree of semantic transparency varies between compounds (compare strawberry or sandman). Our dataset includes both compositional and relatedness-based semantic transparency measures, also differentiated by constituents. The measures are obtained from a computational and fully implemented semantic model based on distributional semantics. We validate the measures using data from four behavioral experiments: Explicit transparency ratings, two different lexical decision tasks using different nonwords, and an eye-tracking study. We demonstrate that different semantic effects emerge in different behavioral tasks, which can only be captured using a multi-dimensional approach to semantic transparency. We further provide the semantic transparency measures derived from the model for a dataset of 40,475 additional German compounds, as well as for 2061 novel German compounds.
\end{abstract}

Keywords Semantic transparency $\cdot$ Lexical decision $\cdot$ Eye tracking $\cdot$ Compound words $\cdot$ Distributional semantics

Many languages make use of compound words such as airport or smartphone in order to express new concepts and meanings (Thagard, 1984). Indeed, compounding is a very prevalent diachronic phenomenon in different languages (Libben, 2014), and is assumed to be central in the development of complex expressions, compositionality and lexical productivity in general (Bauer, 2009; Jackendoff, 2002).

In a complex expression such as a compound, the meanings of the constituents are typically informative about

This work was supported by a Research Fellowship (no. 392225719) from the German Research Foundation (DFG), awarded to Fritz Günther, and by a grant from the German Research Foundation (DFG), awarded to Jens Bölte (BO 1479/8). We thank Andrea Böhl for conducting the studies used to validate our dataset.

Fritz Günther

fritz.guenther@uni-tuebingen.de

1 University of Milano-Bicocca, Milan, Italy

2 NeuroMI, Milan Center for Neuroscience, Milan, Italy

3 Westfälische Wilhelms-Universität Münster, Münster, Germany the meaning of the expression as a whole: An airport shares some semantics with both air and with port. In Germanic languages such as English or German, the rightmost (last) constituent generally serves as the head (Williams, 1981) which defines the semantic category of the word, while the first constituent serves as a modifier further specifying the meaning - an airport is a kind of port dedicated to travel by air. This compositionality of meaning is what makes complex expressions useful in the first place, and allows speakers of a language to create and understand with no effort compounds they have never encountered before (Downing, 1977). Naturally, the role played by the constituents in the comprehension and processing of complex expressions has received a lot of scientific interest.

\section{Semantic transparency}

When investigating semantic effects of the constituents in compound processing, research has relied heavily on the concept of semantic transparency (Marelli et al., 2015; Schäfer, 2018). Compounds such as airport, where the compound meaning is clearly characterized by its constituent meanings, are semantically transparent; on the other hand, compounds such as ladybird, with unclear 


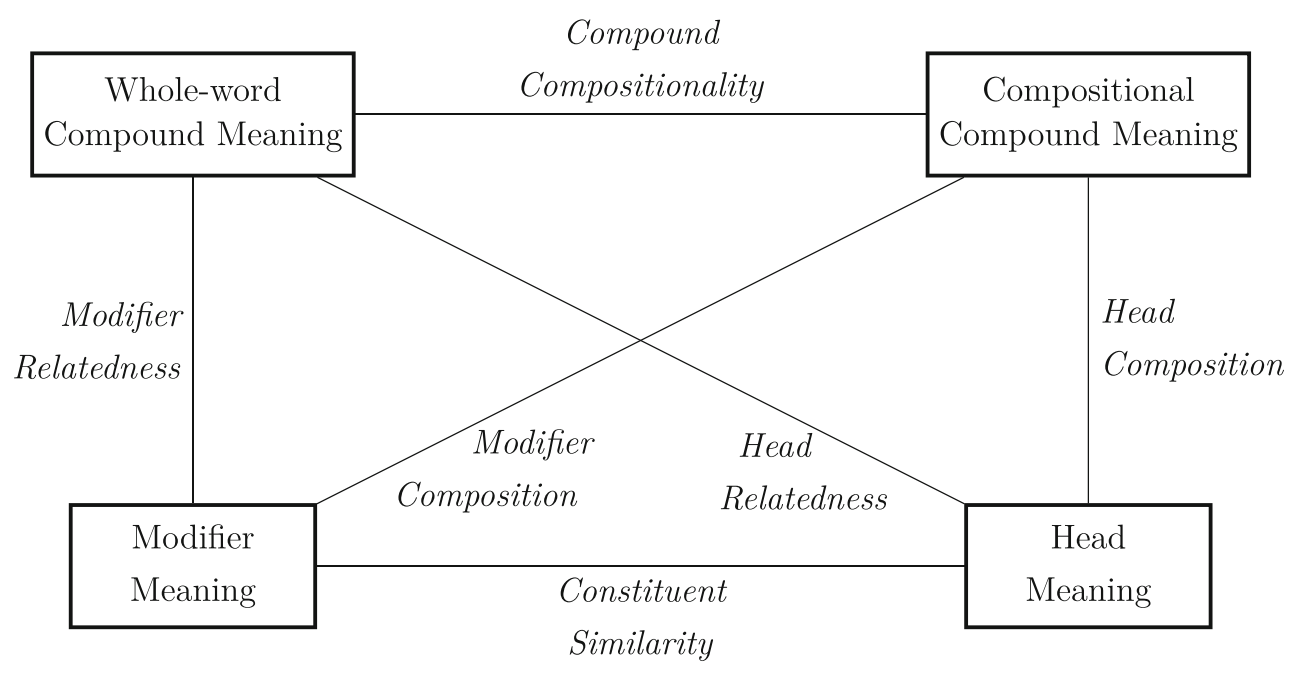

Fig. 1 The model of semantic transparency examined in the present study. In our implementation, the meanings (boxes) are represented as distributional vectors, and the semantic similarities (lines) as cosine similarities. Figure adapted from Günther and Marelli (2018a)

relations to their constituent meanings, are semantically opaque. However, there are (at least) two different ways of defining semantic transparency (see Günther and Marelli (2018a), Marelli and Luzzatti (2012), and Schäfer (2018)). The traditional approach, employed by most psycholinguistic studies to investigate transparency effects, is to define it as the semantic relatedness between the compounds and its constituents (e.g. Sandra (1990), Schmidtke et al. (2018), and Zwitserlood (1994); see Fig. 1). From a psychological point of view, this approach considers the compound and its constituents as separate, distinct units in semantic memory, with activation or inhibition spreading between them (in line with models of semantic memory such as those proposed by Collins and Loftus (1975), Kintsch (1988), and Seidenberg and McClelland (1989)). Semantic relatedness in this view corresponds to the association between these units. In this perspective compounds could be characterized as lexical strings that just happen to embed two other existing words, without considering that they actually describe a single new concept, formed from the combination of its constituent meanings.

In contrast to this relatedness-based perspective, the compositional perspective on semantic transparency highlights the role of the constituents as the building blocks of an active combinatorial process that is aimed at obtaining a compositional compound meaning (see Gagné and Shoben (1997) and Gagné and Spalding (2009)). In this perspective, compounds can be considered as transparent if the constituents strongly contribute to the compositional process, and are easily integrated into the combined meaning, and if their compositionally computed meaning is consistent with a diachronically developed, lexicalized one (Günther \& Marelli, 2016, 2018a; Gagné \& Spalding, 2009).
In this compositional perspective, semantic transparency is therefore inherently linked with interpretability-a property not only describing lexicalized, but also novel compounds. For example, take the German word Angsthase (fraidy-cat, lit. fearbunny). This word is usually considered a semantically opaque compound, because it describes a coward rather than an actual bunny. However, the meaning of Angsthase is still intuitively very predictable, and could easily be interpreted from its constituents even if one encountered it for the very first time, in the same sense as for other, novel combinations: One could as easily refer to someone as an Angstmaus (fearmouse), ${ }^{1}$ or, as suggested by the English translation, an Angstkatze (fraidy-cat). In fact, this predictability is a hallmark and, to some degree, a necessary property of productive systems in general (see also Marelli and Baroni (2015)), of which the English and German compounding systems are examples. In the case of novel compounds, this meaning predictability cannot be due to semantic relatedness between the constituents and the whole-word meaning, or even between a compositional and the whole-word compound meaning, simply because no such whole-word meaning is available when encountering a word for the first time. Rather, these words appear interpretable due to the contributions of their constituents (note that, in the case of fearmouse, we cannot simply claim that only the modifier contributes to the meaningfeareagle or fearlion would not work nearly as good). We will refer to these constituent contributions as modifier composition and head composition in the present article (see Fig. 1).

\footnotetext{
${ }^{1}$ We thank an anonymous reviewer for suggesting this example
} 


\section{Semantic transparency in psycholinguistic research}

A straightforward hypothesis concerning semantic effects of constituents in on-line compound processing is that semantically transparent compounds should have a processing advantage over opaques ones, since their meaning can be accessed via their constituent meanings (Libben, 1998). In studies investigating this hypothesis, there has been a tendency of finding semantic transparency effects on processing times when the latter is conceptualized from a compositional rather than relatedness perspective (Ji et al., 2011; Marelli \& Luzzatti, 2012). In studies not explicitly making this distinction, results have been quite inconsistent across different tasks: While some studies found processing advantages for transparent compounds (priming: Osgood and Hoosain (1974) and Sandra (1990); lexical decision: Bronk et al. (2013) and Libben et al. (2003); gaze durations in eye tracking: Juhasz (2007) and Underwood et al. (1990)), others did not find differences between the two types (priming: Libben et al. (2003) and Monsell (1985); lexical decision: Ji et al. (2011), Experiment 1; eye tracking: Frisson et al. (2008), Experiment 1; Juhasz (2016) and Pollatsek and Hyönä (2005)). This demonstrates that a comprehensive definition and conceptualization of semantic transparency in its different aspects is necessary in empirical investigations of semantic effects in compound processing and comprehension.

To address this issue, Günther and Marelli (2018a) recently proposed a fully implemented computational model based on distributional semantics (for overviews on distributional semantic models, see Günther et al. (2019), Landauer and Dumais (1997), and Turney and Pantel (2010)) to directly measure the different aspects of semantic transparency, both in terms of relatedness and compositionality (see Fig. 1). Distributional semantic models represent word meanings as high-dimensional vectors automatically extracted from large corpora of natural text, based on words' global co-occurrence patterns. These models were already used in many studies (see Schmidtke et al. (2018)) to measure semantic relatedness as the similarities between wholeword compound and constituent vectors, which can both be obtained directly from text corpora. However, measuring semantic transparency in compositional terms requires a representation of a compositional compound meaning as a distributional vector, which has only been made possible recently with compositional distributional semantic models (Marelli et al., 2017). These models are based on a regressional approach trained to optimally predict observed compound meanings from its constituent meanings (both represented as vectors extracted from the corpus; Guevara (2010)). The parameter matrices obtained through training can then be used to induce predicted, compositional meanings for any combination of constituents (see Marelli et al. (2017) and Günther and Marelli (2018a), for technical details).

Using this framework, Günther and Marelli (2018a) investigated semantic effects in simple lexical decision times for a large set of English compounds (Balota et al., 2007). In their study, they found that compositional measures of semantic transparency, rather than relatedness measures, predicted lexical decision times, supporting the assumption that compound processing always includes a compositional process aimed at combining the constituent meanings. This process is facilitated and response times are shorter when the constituents contribute highly to the combined meaning (Gagné \& Spalding, 2009).

\section{The present dataset and related work}

In the present study, we provide and validate a dataset of six different semantic transparency measures (represented by the links in Fig. 1) for a large set of German compounds. For English, different datasets containing human ratings of semantic transparency are available: Juhasz et al. (2015) provide ratings for 629 English compounds, where participants had to indicate whether the compound meaning was transparently related to its constituents (i.e., one single variable per compound). Kim et al. (2018) collected ratings for the transparency of both modifier and head for a set of 2861 English compounds (i.e., two transparency variables per compound). Also, in a very recent study, Gagné et al. (in press) provide a large database of 8376 English compounds, including head and modifier relatedness measures as well as ratings for the extent to which the constituents retain their meaning in the compound, and to which the meaning of the compound is predictable from its parts. Furthermore, Günther and Marelli (2018a) provide a dataset of the semantic transparency measures displayed in Fig. 1head and modifier relatedness, head and modifier composition, and compound compositionality-for 1865 English compounds as a Supplemental Material to their study, available at https://doi.org/10.1037/xlm0000677.supp.

However, comparable resources are not readily available for German, even though German is ideally suited for the investigation of semantic effects in compound representation and processing due to its rich and synchronically extremely productive compounding system (Smolka \& Libben, 2017). Additionally, the word formation of compounds in German is extremely consistent: As in English, the head of German compounds is always the rightmost (last) constituent (Williams, 1981). Differently from English, with its open forms such as tree house, German compounds are consistently written as a single string (Baumhaus). Accordingly, many psycholinguistic studies on semantic transparency are being conducted using German compounds (e.g., Böhl (2007), Bronk et al. (2013), Dohmes et al. 
(2004), Gumnior et al. (2006), Koester et al. (2007), Lorenz et al. (2014), Lorenz and Zwitserlood (2014), Sahel et al. (2008), and Smolka and Libben (2017)).

The most comprehensive German resource to date has been the GhoSt-NN dataset (Schulte im Walde et al., 2016), which includes constituent-wise ratings of compositionality for 868 German compounds (i.e., two transparency variables for each compound, either related to its modifier or head), collected from and averaged over 5 to 13 human expert raters (linguists or computational linguists). However, the instruction given to the participants was to provide these ratings on a scale ranging from "definitely semantically opaque" to "definitely semantically transparent", which can be interpreted as evaluating the relatedness between the compound and each of its constituents, amounting to the modifier and head relatedness measures described in Fig. 1 (compare Appendix A for an analysis of the GhoSt-NN dataset).

Importantly, the GhoSt-NN dataset provides only two constituent-wise transparency measures, and does therefore not explicitly distinguish between constituent composition and constituent relatedness as separate constructs (while the description of the dataset suggests the former, the concrete instruction suggests the latter; compare also Appendix A). Furthermore, the GhoSt-NN dataset does not consider other transparency measures such as constituent similarity or compound compositionality. The present study aims at filling this gap, employing the framework proposed by Günther \& Marelli (2018a, see Fig. 1) to compute semantic transparency measures for 1810 German compounds. As described earlier, the framework is fully implemented as a computational model, using recent developments in distributional semantics. We can thus obtain the semantic transparency measures in an automatic and data-driven way, based on large amounts of natural language data, thereby avoiding the loophole of predicting behavioral data using other behavioral data such as human judgments (see Jones et al. (2015) and Westbury (2016)). Adopting this framework comes with three additional advantages. Firstly, it allows to differentiate different aspects of semantic transparency, namely relatedness and compositionality (Günther \& Marelli, 2018a; Marelli \& Luzzatti, 2012), at the same time considering the different constituent roles (Kim et al., 2018; Libben et al., 2003). Secondly, it allows to measure semantic transparency as a graded variable, beyond a simple and coarse distinction between transparent and opaque compounds (Marelli \& Luzzatti, 2012). Thirdly, the framework can easily be generalized to obtain semantic transparency measures for large sets of compounds (see the large dataset provided in the present article, containing these measures for a total of over 42,000 German compounds).
We validate these measures using data from four behavioral experiments by Böhl (2007): Explicit transparency ratings, two different lexical decision tasks using different nonwords, and an eye-tracking study using the boundary technique. We show that different semantic effects emerge in different behavioral tasks, demonstrating the importance of a comprehensive model of semantic transparency that is able to capture the different aspects of the construct.

\section{The semantic transparency measures}

\section{Word material}

The word material employed in this study consists of 1810 German compound words, obtained from a large-scale study by Böhl (2007), also described in Brysbaert et al. (2011). These compounds were originally collected from the German CELEX database (Baayen et al., 1995). For 344 additional compounds used in the original study, semantic measures could not be obtained due to the frequency restrictions described below. Thus, while the item material of all the experimental studies described here consisted of the same 2154 compounds, all our analyses are based on the same subset of 1810 compounds.

\section{Semantic model and measures}

\section{Source corpus}

The source corpus from which distributional vectors were derived was the $\sim 1.7$ billion word deWaC corpus (Baroni et al., 2009), a web corpus established by crawling through a wide range of German websites. The corpus was lemmatized using TreeTagger (Schmid, 1995), and all computations described here were performed at the lemma level.

\section{Semantic space}

Distributional vectors were obtained from this source corpus using the CBOW algorithm of the word2vec model (Mikolov et al., 2013). This algorithm trains distributional vectors as the hidden layer of a neural network predicting the $n$ words surrounding a target word to each side (see Hollis (2017), Mandera et al. (2017), and Mikolov et al. (2013), for technical details). This model has been shown to implement psychologically plausible learning mechanisms for semantic representations (Hollis, 2017; Mandera et al., 2017). Additionally, it has been demonstrated that this model has an excellent performance in a variety of semantic tasks (Baroni et al., 2014) and in predicting human behavior 
Table 1 Compounds scoring very high or very low on relatedness variables

\begin{tabular}{lll}
\hline Variable & Low values & High values \\
\hline Modifier relatedness & Hauptperson (leading character, lit. headperson) & Osterfest (Easter, lit. Eastercelebration) \\
& Steuerbord (starboard, lit. steeringboard) & Enkelkind (grandchild, lit. grandchild-child) \\
Head relatedness & Schmutzfink (litterbug, lit. dirtfinch) & Sandstrand (sand beach, lit. sandbeach) \\
& Feuerwerk (firework, lit. firework) & Hausarzt (family doctor, lit. housephysician) \\
Constituent similarity & Luftlinie (air-line distance, lit. airline) & Barthaar (hair of the beard, lit. beardhair) \\
& Preisschild (pricetag, lit. pricesign) & Seeufer (lakeshore, lit. lakeshore)
\end{tabular}

in experimental paradigms tapping into semantic processing (Mandera et al., 2017).

To set up the model, we used the best-performing parameter set from the study of Baroni et al. (2014): A context window size of 5 words, and a network architecture producing 400-dimensional vectors (negative sampling with $k=10$, subsampling with $t=1 e^{-5}$ ). To obtain reliable representations, we only considered words with a frequency higher than 50 in the source corpus for training the model. The semantic space is available online at https://sites.google.com/site/fritzgntr/ software-resources/semantic_spaces (Günther et al., 2015), labeled dewac_cbow.

With this model, we obtained distributional vectors representing the constituent meanings as well as the wholeword compound meanings for the 1810 compounds in our word material. Using the DISSECT toolkit (Dinu et al., 2013), we then computed the following measures on the basis of these vectors:

- modifier relatedness: the cosine similarity between the modifier vector and the whole-word compound vector, measuring the degree of semantic relatedness between the modifier and the whole-word compound meaning, conceptualized as distinct, separate entries in semantic memory (see Schmidtke et al. (2018)).

- head relatedness: the cosine similarity between the head vector and the whole-word compound vector, analogously to modifier relatedness

- constituent similarity: the cosine similarity between the modifier vector and the head vector, measuring the semantic coherence between the compound constituents (Lynott \& Ramscar, 2001). Concepts that are more consistent should be easier to align and to combine into a new concept.

Examples for compounds scoring high or low on these variables are provided in Table $1 .^{2}$

\footnotetext{
${ }^{2}$ Examples for English compounds are provided by Günther and Marelli (2018a).
}

\section{Compositional model}

In addition to the relatedness measures described above, we computed compositional transparency measures for the compounds in our word material. To this end, a vector representation of the compositional compound meaning is required. In this study, we used the CAOSS model (Marelli et al., 2017), which is based on earlier proposals by Guevara (2010), to obtain these representations (see also Günther and Marelli (2018a)).

The CAOSS model computes a compositional compound meaning as

$c=M \cdot u+H \cdot v$,

where $c$ is the $k$-dimensional vector representing the compound meaning ( $k=400$ in the present study), $u$ and $v$ are the $k$-dimensional vectors representing the left and right constituent, respectively, and $M$ and $H$ are $k \times k$-dimensional weight matrices updating the constituent meanings into specific role-dependent meanings before they are combined.

The free parameters of this model, the weight matrices $M$ and $H$, are obtained in a regression-based training process: With a given training set consisting of compounds and their constituents, $M$ and $H$ are estimated in a leastsquare regression procedure aimed at optimally predicting the whole-word compound meanings for these compounds (derived from the source corpus) from their constituent meanings $u$ and $v$, following Eq. 1. In our study, this training set consisted of 42,285 compounds for which constituent and whole-word compound vectors were available in our semantic space. This training set was collected from the word material used in the present study (Böhl, 2007; Brysbaert et al., 2011), the GhoSt-NN dataset (Schulte im Walde et al., 2016), and a very large compound set from GermaNet v.12 (Henrich \& Hinrichs, 2011). 21,821 additional compounds collected from these sources were not included in the training set because one of the required vector representations was not available in our semantic space. The training was conducted using the DISSECT toolkit (Dinu et al., 2013). 
Table 2 Compounds scoring very high or very low on compositional variables (with more example for cases where relatedness and composition are dissociated)

\begin{tabular}{lll}
\hline Variable & Value & Examples \\
\hline Modifier composition & low & Grundschule (elementary school, lit. groundschool) \\
& & Schulfreund (schoolmate, lit. schoolfriend) \\
Head composition & high, mod. relatedness high & Paradebeispiel (prime example, lit. parade-example) \\
& low & Felswand (rock face, lit. rockwall) \\
& high, head relatedness low & Bankwesen (banking sector, lit. bankbeing) \\
& & Zeitraum (space of time, lit. timespace) \\
Compound compositionality & Sigh, head relatedness high & Stammbaum (family tree, lit. trunktree) \\
& low & Wintersemester (winter semester, lit. wintersemester) \\
& & Zebrastreifen (crosswalk, lit. zebrastripes)
\end{tabular}

Note that there are no items in our dataset that have low composition values but high relatedness values

We then used the weight matrices $M$ and $H$ obtained through training to derive a compositional compound meaning $c$ for all compounds in our word material, following Eq. 1. With these compositional meaning representations, we computed for each compound in the word material:

- modifier composition: the cosine similarity between the modifier vector and the compositional compound vector, measuring the contribution of the modifier meaning to the compositional process and its product, the compositional compound meaning (Günther \& Marelli, 2016), and the ease of integrating the modifier meaning into a combined concept (Gagné \& Spalding, 2009)

- head composition: the cosine similarity between the head vector and the compositional compound vector, analogous to modifier composition

- compound compositionality: the cosine similarity between the whole-word and the compositional compound vector, measuring the meaning predictability of the compound given its constituents (see Plag (2003) and Schäfer (2018))

Examples for compounds scoring high or low on these variables are provided in Table $2 .^{3}$

The dataset containing all the described measures for the 1810 compounds of our word material is provided at https://doi.org/10.6084/m9.figshare.8295101. Due to the limited availability of experimental data, we only validate

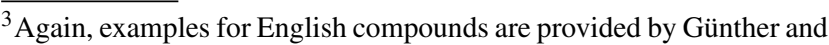
Marelli (2018a).
}

the semantic measures for the 1810 compounds in our word material. However, in principle, our model architecture allows us to compute semantic measures for all compounds included in the training set. We provide the semantic measures for the remaining 40,475 compounds at the same online repository, with the explicit cautionary note that they are not empirically validated.

\section{Lexical variables}

In addition to the semantic measures described here, we also considered several lexical variables as a non-semantic baseline in our subsequent analyses. We opted to include the most prominent variables considered in the literature on compound processing (length, frequencies, family sizes), which are often associated to morphological and lexical rather than semantic processes and representations (e.g. Kuperman et al. (2009)). Frequency measures (modifier frequency and head frequency when used as free words, as well as compound frequency) were obtained directly from the source corpus. Modifier and head family sizedefined as the number of compound types sharing the constituent in question-were collected from our training set of 42,285 compounds. Pairwise correlations between all semantic transparency measures and all frequency and family size measures for our item set are shown in Table 3 .

\section{Validation}

In the following sections, we validate our measures using four behavioral datasets presented in and collected by Böhl 
Table 3 Pairwise correlations between relatedness measures (rel.), compositional measures (comp.), frequencies (freq.), and family sizes (fam.) for compounds (c.), their modifiers (m.) and their heads (h.)

\begin{tabular}{|c|c|c|c|c|c|c|c|c|c|c|}
\hline . & h.rel. & const.sim & m.comp. & h.comp. & c.comp. & m.freq. & h.freq. & c.freq & fam.m. & fam.h. \\
\hline m.rel. & 0.04 & 0.46 & 0.62 & 0.04 & 0.58 & -0.03 & 0.03 & 0.12 & 0.00 & 0.06 \\
\hline h.rel. & & 0.42 & 0.02 & 0.60 & 0.62 & -0.02 & -0.12 & 0.14 & 0.01 & -0.18 \\
\hline const.sim. & & & 0.59 & 0.56 & 0.34 & -0.10 & -0.16 & -0.01 & -0.07 & -0.20 \\
\hline m.comp. & & & & -0.10 & 0.22 & -0.12 & 0.01 & -0.08 & -0.02 & -0.02 \\
\hline h.comp & & & & & 0.29 & 0.00 & -0.19 & -0.01 & -0.07 & -0.25 \\
\hline c.comp. & & & & & & 0.01 & -0.01 & 0.27 & 0.09 & 0.02 \\
\hline m.freq. & & & & - & & & 0.18 & 0.32 & 0.62 & 0.12 \\
\hline h.freq. & & & & & & & & 0.34 & 0.11 & 0.68 \\
\hline c.freq. & & & & & & & & & 0.26 & 0.32 \\
\hline fam.m. & & & & & & & & & & 0.08 \\
\hline
\end{tabular}

Constituent similarity is abbreviated as const.sim. Correlations that are significantly different from zero are displayed in boldface

(2007): Explicit ratings on semantic transparency, two different lexical decision tasks using different sets of nonwords, and an eye-tracking study using the boundary technique, in which participants have to give semantic relatedness judgments after reading the target compound (Beauvillain, 1996; Rayner, 1975). In the present study, we present brief summaries of the experimental methodology; detailed descriptions including participants, material, and procedure are provided by Böhl (2007). We investigated whether semantic effects can be detected in all four paradigms, using the semantic transparency measures presented here. We further investigated whether different semantic effects emerge in different experimental paradigms, adopting the detailed, explicit and fully implemented model of semantic transparency postulated by Günther and Marelli (2018a).

Our general expectation was that measures depending on the whole-word compound meaning-such as constituent relatedness - should predict behavioral data in tasks where access to this "actual", lexicalized word meaning is necessary-first and foremost, explicit ratings on this very meaning (see Marelli and Baroni (2015)). On the other hand, based on previous results (Günther and Marelli (2018a); see also Marelli and Baroni (2015)), we expected that measures depending on the compositional compound meaning - such as constituent composition-should predict on-line processing times in lexical decisions, where access to the actual word meanings is not necessarily required by the task. However, in these two paradigms, the task demands for semantic access to the whole-word meaning directly coincide with the mode of measurement (on-line vs. offline task). To disentangle how these variables affect the influence of the measures investigated here, the boundary technique provides an interesting test case as an on-line processing tasks that requires access to the actual compound meaning. In this case, we have no clear predictions based on previous studies, but would assume that the role of the whole-word meaning access on the one hand and the compositional process on the other hand depends more on the task demands (shallow lexical access as required for lexical decision vs. semantic access as required for full meaning retrieval) than the mode of measurement (on-line vs. off-line task). If that is the case, measures derived from the whole-word meaning should be more predictive here than compositional measures.

In each analysis, we started from a baseline model containing orthographic length, log-transformed constituent and compound frequencies, and family sizes, as well as random intercepts for modifiers and heads. Then, in a first screening step, we tested which of the semantic transparency measures significantly improves these baseline models in a likelihood-ratio test. This analysis was conducted to identify which semantic effects can be expected in the different paradigms when they are tested in isolation.

Finally, we conducted a stepwise forward model analysis to identify the set of parameters that optimally predicts each dataset. In each step, we tested separately for each semantic parameter not yet included in the model whether it significantly improved the current model, as indicated by a likelihood-ratio test. In cases where more than one possible candidate significantly improved the current model, we selected and included the best-predicting parameter (for which the resulting model has the lowest AIC value; Akaike (1998)). This was repeated until no further parameter significantly improved the model. Applying this procedure allows us to deal with the collinearity of the predictor variables (see Table 3): In each likelihood-ratio test, two models are compared, one containing all the variables included so far, and one additionally containing one extra parameter. This parameter is only added to 
the simpler model if the resulting more complex model has a significantly higher model likelihood, and thus if the additional parameter significantly improves the model predictions over and above the parameters already included in the model. Since variance shared between a new predictor and correlated predictors already included in the model cannot improve the model predictions, only parameters explaining additional variance of the dependent variable will be included. ${ }^{4}$

To exclude the impact of overly influential outliers in the final models, data points were removed on the basis of a threshold of 2.5 SD standardized residual errors after having defined the models, and the models were then re-fitted on the truncated dataset (model criticism; Baayen (2008)). All frequency estimates, response times in the lexical decision tasks, as well as first-fixation durations and gaze durations in the eye-tracking study were log-transformed (Baayen \& Milin, 2010). All analyses were conducted using the packages lme4 (Bates et al., 2015) and lmerTest (Kuznetsova et al., 2017) for R (R Core Team, 2017).

\section{Explicit ratings of semantic transparency}

For all the compounds in the word material, Böhl (2007) collected and aggregated explicit semantic transparency ratings from 26 native German speaking participants. Participants were first instructed about the concept of semantic transparency, stating that the meaning of transparent compounds (such as Feldhase, hare, lit. field hare) can be inferred from their constituents, but that the meaning of opaque compounds (Angsthase, fraidy-cat, lit. fear hare) cannot be derived from their constituents. They were then asked to "judge the combined words (compounds) regarding their semantic transparency", on a scale from 1 (semantically transparent) to 5 (semantically opaque). Each participant gave ratings for all 2154 compounds, split into four different pen-and-paper questionnaires. Participants would be handed the next questionnaire (and could take it with them) after submitting the previous one. The estimated time to fill in each of the four questionnaires was two hours.

\section{Analysis and discussion}

Since modifier frequency, compound frequency, and head family size did not significantly predict rating scores, these parameters were eliminated from the baseline model. In the

\footnotetext{
${ }^{4}$ Of course, the model parameter estimates in the resulting final models are still affected by collinearity, since these are all estimated simultaneously. However, the alternative solution-de-correlating the variables to report parameter estimates in the final model-would also leave us with estimates that are very hard to interpret. Therefore, we opted to report the estimates for the variables as they are.
}

Table 4 Results of the likelihood ratio tests testing whether the semantic measures on their own improve the baseline model for explicit semantic transparency ratings

\begin{tabular}{lllll}
\hline Parameter & $b$ & $t$ & $X^{2}(1)$ & $p$ \\
\hline Modifier relatedness & -2.20 & -14.32 & 194.32 & $<.001$ \\
Head relatedness & -1.87 & -12.83 & 156.82 & $<.001$ \\
Constituent similarity & -2.26 & -14.79 & 205.65 & $<.001$ \\
Modifier composition & -2.27 & -9.90 & 94.50 & $<.001$ \\
Head composition & -2.68 & -11.42 & 125.04 & $<.001$ \\
Compound compositionality & -2.78 & -16.77 & 269.52 & $<.001$ \\
\hline
\end{tabular}

first screening step, all six semantic transparency measures on their own significantly improved the baseline model (see Table 4). Note again that Table 4 displays the results of six different parallel analyses, all with reference to the baseline model.

The final model, after forward selection, includes most, but not all of the semantic parameters: No parameter for modifier composition was added in the stepwise procedure. The parameters of the final model after model criticism are displayed in Table 5. As can be seen in Tables 4 and 5, for all semantic transparency measures higher values predict lower rating scores, which correspond to more transparent compounds.

As can be seen from the screening analysis, each semantic transparency measure on its own predicts transparency ratings very well. This supports the general validity of our computationally derived similarities between distributional vectors as psychologically plausible measures of semantic transparency.

Furthermore, our forward model selection shows that almost all our measures independently add predictive power in modeling semantic transparency ratings. Firstly, this confirms that they indeed capture different and independent aspects of semantic transparency. Secondly, it shows that

Table 5 Parameters for the final model optimally predicting explicit semantic transparency ratings, after model criticism. Parameters for semantic measures are listed in order of their inclusion in the model

\begin{tabular}{lllll}
\hline Step & Parameter & $b$ & $t$ & $p$ \\
\hline & intercept & 5.07 & 19.18 & $<.001$ \\
& length & -0.03 & -2.24 & .026 \\
& head frequency & -0.06 & -4.62 & $<.001$ \\
& modifier family size & -0.01 & -2.15 & .032 \\
1. & compound compositionality & -1.21 & -4.10 & $<.001$ \\
2. & constituent similarity & -0.90 & -4.25 & $<.001$ \\
3. & modifier relatedness & -0.88 & -3.75 & $<.001$ \\
4. & head composition & -0.65 & -2.17 & .018 \\
5. & head relatedness & -0.55 & -2.32 & .021 \\
\hline
\end{tabular}


human ratings of semantic transparency, especially when given as a single overall rating, are an amalgamation of different semantic aspects, which can be separated using an appropriate and detailed model of semantic transparency. Interestingly, when no specific instructions are provided in the ratings task i.e., whether to judge relatedness or compositionality, human judgments seem to be influenced both by compositional aspects (such as compound compositionality, corresponding to meaning predictability) and constituent-wise relatedness.

\section{Lexical decision tasks}

\section{Experiment 1: Word-nonword combinations as nonwords}

In a lexical decision task, participants are presented with character strings and have to decide whether these strings are actual words or not. In order to perform a lexical decision, participants are assumed to rely on cues provided by the stimuli-such as orthographic, but also semantic information-that allow them to reliably discriminate between existing and non-existing words (Seidenberg \& McClelland, 1989). Semantic effects can be expected to influence performance in this task, since real words should lead to larger semantic activation than nonwords, leading to shorter response times.

In the material employed by Böhl (2007), the nonwords were created from the original item material by changing the first, middle, or final grapheme in one of the constituents for each item. Thus, the target words in the material were the original 2154 real compound words (e.g., Flughafen, airport), while the 2154 nonwords consisted of one real-word constituent and one nonword constituent (e.g., Flughafan, airpurt). Nonwords were created this way in order to ensure the highest possible form similarity to the target words, and therefore to minimize confounding (for example, the impact of orthographic cues; Seidenberg and McClelland (1989)).

Experimental data were collected from 16 native German speakers. The material was split into two lists, each containing half of the actual-word stimuli from the word material, and the other half in their nonword versions. Each participant was presented with one of these lists, divided into eight experimental blocks. Participants were instructed to indicate whether the letter strings presented to them where actual, existing German words. The stimuli appeared after a fixation cross. The "yes"-answers to the lexical decision task were given by pressing the right button on a two-button response divide, and the "no"-answers by pressing the left button. For more details on the data collection, see Böhl (2007).
Error trials and trials with response times larger than $1600 \mathrm{~ms}$ were removed before the data were aggregated over participants (Böhl, 2007). In this and the following experiments, the relatively small number of participants (eight data points per item) was counter-balanced by the very large number of items employed, basing each study on quite a large number of datapoints. Critically, since we employ several continuous predictors that are inherently tied to the items, it is this large number of items which allows us to reliably validate our measures.

\section{Analysis and discussion}

The parameter for head frequency was removed from the baseline model as it did not significantly predict response times.

In the first screening step, the inclusion of three parameters on their own significantly improved the baseline model: modifier composition $\left(X^{2}(1)=9.30, p=.002, b=\right.$ $-0.09, t=-3.07)$, head composition $\left(X^{2}(1)=4.01, p=\right.$ $.045, b=-0.07, t=-2.01)$, and constituent similarity $\left(X^{2}(1)=9.18, p=.002, b=-0.07, t=-3.05\right)$.

The final model after forward selection contains parameters for both modifier composition and head composition, but not for constituent similarity. Both parameters are negative, so higher values predict faster response times. The parameters of the final model after model criticism are displayed in Table 6.

The results of this analysis are very similar to the results obtained in English by Günther and Marelli (2018a), who also observed facilitatory effects of modifier and head composition in a lexical decision paradigm, but no effect

Table 6 Parameters for the final model optimally predicting response times in the lexical decision experiments, after model criticism. Parameters for semantic measures are listed in order of their inclusion in the model

\begin{tabular}{llllll}
\hline Experiment & Step & Parameter & $b$ & $t$ & $p$ \\
\hline Exp. 1 & & intercept & 6.77 & 175.57 & $<.001$ \\
& & length & -0.03 & 14.46 & $<.001$ \\
& & modifier frequency & -0.01 & -4.83 & $<.001$ \\
& & compound frequency & -0.03 & -14.51 & $<.001$ \\
& 1. & modifier composition & -0.08 & -2.73 & .007 \\
& 2. & head composition & -0.07 & -2.22 & .027 \\
Exp. 2 & & intercept & 6.83 & 189.15 & $<.001$ \\
(targets) & & length & -0.02 & 10.52 & $<.001$ \\
& & head frequency & 0.01 & 3.93 & $<.001$ \\
& & compound frequency & -0.04 & -19.52 & $<.001$ \\
& 1. & modifier composition & -0.06 & -1.99 & .047 \\
Exp.2 & & intercept & 6.61 & 416.57 & $<.001$ \\
(nonwords) & & length & 0.04 & 21.10 & $<.001$ \\
& 1. & modifier composition & 0.04 & 2.11 & .035 \\
\hline
\end{tabular}


of relatedness measures. Thus, we replicate these previous findings, despite the differences between the material at hand: The data examined by Günther and Marelli (2018a) were part of the English Lexicon Project, so only some of the words included were compounds, and the vast majority of nonwords did not have a constituent structure including one orthographically valid constituent. Notably, we observe the same pattern in German, serving as a cross-linguistic validation of the semantic model (Marelli et al., 2017) and the measures derived from it.

This generalizes the findings by Günther and Marelli (2018a), supporting the assumption that compound processing, as measured by lexical decision, involves an automatic compositional process combining the constituent meanings into a single concept (Gagné \& Shoben, 1997; Gagné \& Spalding, 2009). Taken together with these previous findings, the present results show that-at least in the case of lexical decision tasks - the semantic processing of compounds should be investigated from a compositional rather than a relatedness perspective. Thus, our findings further validate the measures included in our present dataset, and highlight the importance of compositional measures of semantic transparency.

\section{Experiment 2: Word-word combinations as nonwords}

In Experiment 1, nonwords were constructed by combining an actual compound constituent with a non-existing character string. In the second lexical decision experiment, a different set of nonwords was employed, by combining two existing words into a non-existing compound, such as Knotenpferd (knot horse) or Ritterglocke (knight bell). These were created by changing one of the constituents of the original item material. Thus, the nonwords in this task can also be classified as novel compounds, for which a (more or less sensible) interpretation can often be derived due to the synchronical productivity of the German compounding system. However, they differ from actual compounds in that participants can be expected to not have encountered them before, and therefore not to have an established whole-word meaning representation for them.

Except for the nonwords, this second lexical decision experiment was identical to the first one. Data were collected from 16 native German speaking participants. Again, each participant was presented with half of the actual-word stimuli and half of the nonword stimuli from the word material (for more details, see Böhl (2007)).

\section{Target words}

Error trials and trials with response times larger than 1600 ms were again removed before the data were aggregated over participants. The parameter for modifier frequency did not significantly predict response times and was thus removed from the baseline model.

In the first screening step, only the inclusion of modifier composition significantly improved the baseline model $\left(X^{2}(1)=5.85, p=.016, b=-0.08, t=-2.44\right)$. Accordingly, the final model after forward selection (see Table 6) only contains modifier composition as a semantic parameter. The parameter value is negative, indicating faster response times for higher values of modifier composition.

\section{Nonwords}

As mentioned previously, the nonwords in this experiments can be described as novel compounds, for which a meaning can be derived in a compositional procedure (Marelli et al., 2017). This allows us to investigate semantic effects in the processing of these novel compounds, using the framework outlined by Günther and Marelli (2018a). Note however that no vector representing the whole-word compound meaning is available for these compounds. Thus, semantic measures that depend on the whole-word meaning (relatedness measures and compound compositionality) cannot be considered, leaving modifier composition, head composition, and constituent similarity.

Using the CAOSS model and the same parameter matrices as for the word material, we computed these measures for 2061 out of the 2154 novel compounds used in this study (due to the frequency restrictions, the measures could not be computed for the remaining 93 compounds). The measures are provided at https://doi.org/10.6084/m9. figshare.8295101, along with the other datasets. Error trials (that is, trials in which participants erroneously responded that the compound presented to them is an existing word) and trials with response times larger than $1600 \mathrm{~ms}$ were again removed before the data were aggregated over participants.

Since the compound frequency for all the compounds is zero, the parameter was not included in the baseline model. Furthermore, modifier and head frequency both did not predict reaction times and were not included in the baseline model.

In the first screening step, there only was a borderline effect of modifier composition $\left(X^{2}(1)=3.80, p=\right.$ $.051, b=0.04, t=1.96$ ), but no effect of head composition or constituent similarity. However, after the removal of overly influential data points via model criticism, the final model contained a significant effect of modifier composition (see Table 6). Here, the parameter value is positive, indicating slower response times (i.e., slower rejections of the compound as non-existing) for higher values of modifier composition. 


\section{Discussion}

In the analysis of the target words, we again observed an effect of modifier composition in compound processing, as measured in a lexical decision paradigm (in fact, we only observed this one effect). Again, we find no effects of relatedness measures.

This is in line with the findings by Günther and Marelli (2018a) and the results of the first lexical decision experiment reported here. Unlike these previous experiments, we did not find an effect of head composition for this second lexical decision experiment. However, this can be explained within the framework of theories postulating an automatic combinatorial process during compound processing (Gagné \& Shoben, 1997; Marelli et al., 2017; Spalding et al., 2010). In these theories, the role of the modifier in the compositional process is to make available possible semantic relations connecting it to the head, which are then evaluated against both modifier and head meanings. Thus, a more prominent and active role in guiding the compositional process is ascribed to the modifier. This was already discussed in Günther and Marelli (2018a), who found a stronger effect for modifier composition than for head composition-a pattern which also emerged in Experiment 1. Thus, since the modifier guides the compositional process, and in this task a compositional meaning can be derived both for words and nonwords, the degree of the modifier's ease of composition becomes the most important determinant of processing.

The facilitatory effect of modifier composition in the target words is reversed for the nonwords: Participants take longer to reject novel compounds when the modifier is more easily integrated into a compositional compound meaning. In addition, it has been shown that constituent compositions are major predictors for the plausibility of (novel) compounds (Günther \& Marelli, 2016), and can thus be assumed to measure the semantic interpretability of such novel combinations. Taken together, this indicates that participants have more difficulties in correctly classifying as non-existent compounds which are perceived as better actual-word candidates. These results complement the findings for the target words, and further corroborate the view that compound processing (as measures in lexical decision) entails an automatic, obligatory compositional process guided by the modifier (Gagné \& Spalding, 2009).

\section{Eye tracking with the boundary technique}

The final validation experiment contains data collected in an eye-tracking study with the boundary technique (Beauvillain, 1996; Rayner, 1975). In this experimental paradigm, participants have to read a word presented on a screen and understand its meaning. To ensure that the participants actually access the word meanings, participants then have to read a second word (which could be a noun, verb or adjective) and decide whether the two words are semantically related.

The data for this experiment were collected from eight participants, who each were presented with the full set of word material. The items were presented to the participants in four experimental sessions à four blocks each. In each trial of this experiment, a fixation cross was first presented at the left margin of the screen (100 pixels to the right of the left margin). After participants fixated the cross, both words were presented simultaneously, with the target word on the left and the second word on the right hand side of the screen. The second word was masked with hashes, which were removed once the target word has been read and participants made a saccade across an invisible boundary between the two words. At that point, the target word was masked and so remained until the end of the trial. Participants then had to decide whether the two words were related. For more details on the experimental procedure, see Böhl (2007).

First fixation durations and gaze durations (defined as the sum of all fixations on the target word) were collected. First fixation durations lower than $70 \mathrm{~ms}$ were removed from the data, as were gaze durations lower than $200 \mathrm{~ms}$ or higher than $1600 \mathrm{~ms}$ and trials with incorrect answers in the semantic relatedness judgments (following Böhl (2007)), before the data were aggregated over participants.

\section{First fixation durations}

Parameters for head frequency and modifier frequency did not predict first fixation durations and were thus not included in the baseline model.

In the first screening step, only the inclusion of modifier relatedness significantly improved the baseline model (see Table 7). The final model after forward selection also only contains a parameter for modifier relatedness. ${ }^{5}$ The parameter value is positive, indicating longer first fixations the more similar the modifier meaning is to the whole-word compound meaning.

\section{Gaze durations}

For gaze durations, the parameter for head frequency did not significantly predict response times and was thus not included in the baseline model.

In the first screening step, the inclusion of head relatedness, modifier composition, constituent similarity, and

\footnotetext{
${ }^{5}$ This is not due to the fact that for longer compounds the whole word (and therefore the head) potentially can't be fully read during first fixations: In an control analysis, no interaction between any semantic parameter and length entered the model
} 
Table 7 Parameters for the final model optimally predicting first fixation durations and gaze durations in the eye-tracking paradigm with the boundary technique, after model criticism

\begin{tabular}{|c|c|c|c|c|c|}
\hline Dependent measure & Step & Parameter & $b$ & $t$ & $p$ \\
\hline \multirow[t]{4}{*}{ First fixation } & & intercept & 5.59 & 287.55 & $<.001$ \\
\hline & & length & -0.02 & -14.43 & $<.001$ \\
\hline & & compound frequency & -0.004 & -2.45 & .014 \\
\hline & 1. & modifier relatedness & 0.05 & 2.68 & .007 \\
\hline \multirow[t]{6}{*}{ Gaze duration } & & intercept & 5.87 & 178.61 & $<.001$ \\
\hline & & length & 0.05 & 28.28 & $<.001$ \\
\hline & & modifier frequency & -0.01 & -6.66 & $<.001$ \\
\hline & & compound frequency & -0.02 & -11.49 & $<.001$ \\
\hline & 1. & head relatedness & -0.06 & -2.91 & .004 \\
\hline & 2. & modifier composition & -0.05 & -1.81 & .070 \\
\hline
\end{tabular}

Fore gaze durations, the parameter for modifier composition added in the forward selection is no longer significant after model criticism

compound compositionality on their own each improved the baseline model (see Table 8). The final model, after removal of influential data points by model criticism, however only contains a significant parameter for head relatedness; although a parameter for modifier composition was added to the model in the second step of the forward analysis, the parameter was non-significant after model criticism (see Table 7). The parameter value for head relatedness is negative, indicating shorter gaze durations for higher values of head relatedness.

\section{Discussion}

In the eye-tracking data, we obtain a very different pattern of results compared to the lexical decision experiments. Most notably, we do not observe an effect of compositional measures of semantic transparency. Instead, we observe effects of the semantic relatedness between constituents and the whole-word compound meaning.

A critical difference between the task employed here and the lexical decision task of the previously presented lexical decision experiments was that participants had to access the actual meaning of the whole-word compound in order to perform the subsequent semantic relatedness judgment. In contrast, in a lexical decision task, participants

Table 8 Results of the likelihood ratio tests testing whether the semantic measures on their own improve the baseline model for gaze durations

\begin{tabular}{lllll}
\hline Parameter & $b$ & $t$ & $X^{2}(1)$ & $p$ \\
\hline Head relatedness & -0.06 & -2.79 & 7.72 & .005 \\
Modifier composition & -0.07 & -2.32 & 5.39 & .020 \\
Constituent similarity & -0.05 & -2.30 & 5.35 & .021 \\
Compound compositionality & -0.07 & -2.73 & 7.42 & .006 \\
\hline
\end{tabular}

are only required to identify as fast as possible whether a string presented to them is an existing word or not. For lexical decision, semantic activation serves as a cue for the "wordedness" of the character string (Seidenberg \& McClelland, 1989), but the actual meaning of the string is not particularly relevant. This is different in a semanticrelatedness evaluation, where accessing the actual wholeword compound meaning is necessary in order to properly perform the task. Thus, a compositional process in which a compositional compound meaning is easily obtained would result in faster lexical decisions, but not necessarily in faster reading for comprehension.

The facilitatory head relatedness effect observed in gaze durations is in line with this argumentation: The head defines the semantic category of a compound word (Williams, 1981): A firetruck is a kind of truck. The participants' task in this experimental paradigm was to judge whether the target word and a subsequently presented word were semantically related. To achieve this, they have to access the actual, whole-word compound meaning of the target word, which includes the semantic category to which it belongs (i.e., they have to "understand what the word is"). This is facilitated in cases where this information is congruent, and where the head is highly informative about the compound meaning; that is, for compounds with a high head relatedness.

In first fixation durations, and thus in early processing (Rayner, 1998) we instead observe an effect of modifier relatedness (compare also Schmidtke and Kuperman (2019), for similar findings). Since the fixation cross is presented at the left of the screen, and participants are used to reading German words from left to right, it is expected that they first fixate and access the modifier (the left constituent in German). Therefore, unlike the head, the modifier (and its meaning) are accessed already in the first fixation and affect first fixation durations (Hyönä et al., 2005; Kuperman 
et al., 2009). Additionally, Kuperman et al. (2009) show that whole compound is already accessed in the first fixation, and discuss that this may be attributed to semantic access. Given these findings, the semantic relatedness between modifier and head can already affect early processing, as also demonstrated in a very recent study on English compounds by Schmidtke and Kuperman (2019).

However, the direction of the modifier relatedness effect (being inhibitory) is less straightforward to explain. The most likely explanation for this pattern is a competition for activation between the modifier meaning and the whole-word compound meaning, as thoroughly discussed by Schmidtke et al. (2018). In this case, more similar meanings are more difficult to discriminate, resulting in slower processing. Unlike the head, the modifier does not specify the semantic category of the compound, but only some meaning aspects (an airport is something quite different from air, even if it is highly related to air). Therefore, a highly related modifier meaning can be misleading when the task is to access the actual compound meaning, while a highly related head usually is informative of this actual meaning.

\section{General discussion}

In the present study, we provide semantic transparency measures for 1810 German compounds, on the basis of a computational model implemented in the distributional semantics framework. These measures are validated in four behavioral experiments. We also provide a large dataset containing these measures for 40,475 additional compounds and 2061 nonwords (novel compounds), which might be useful in future studies.

\section{Practical applications}

The practical uses for the datasets provided in the present study are manifold: On the one hand, the included semantic transparency measures can be used to investigate, or control for, semantic effects in new psycholinguistic studies, or to thoroughly examine such effects in existing studies and datasets. Furthermore, they can be used as quantitative reference points in order to empirically test the predictions of linguistic theories on the semantics of compounding. On the other hand, the included behavioral measuresratings, lexical decision response times, and eye-tracking measures-can serve as a gold standard for the evaluation of new models and theories on compounding, with the semantic measures provided here serving as a reference benchmark.

In combination with the English resources already available in the literature (especially with the set by Günther and Marelli (2018a), providing comparable computational measures), the present dataset can also be applied to investigate cross-linguistic issues. One possible question is whether there are structural differences between the semantics of English and German, which can result in differences concerning their representation and processing (see Günther et al. (2019), for such an approach in the domain of affixed words, also applying relatedness and compositionality measures derived from distributional semantic models). Conversely, such studies can also investigate general cross-linguistic similarities in productive combinatorial systems such as compounding (see Günther and Marelli (2018b)).

\section{Theoretical implications}

We demonstrated that our semantic transparency measures predict behavioral data across different experimental paradigms, including explicit ratings, different lexical decision tasks, and data from an eye-tracking paradigm. In general, these results show that the constituent meanings play an important role in the comprehension and processing of compounds.

Importantly, different semantic measures predict behavioral data in different experimental paradigms. Thus, the role played by the constituent meanings is dependent on the task at hand. On the one hand, this shows that our measures are able to detect different semantic influences on compound processing and comprehension. On the other hand, it demonstrates that selecting an adequate task to investigate specific semantic effects is a crucial factor in studies on this topic - which offers an explanation for the inconsistent findings in the literature, which used a wide range of tasks and for the most part non-differentiated conceptualizations and measures of semantic transparency.

Our results suggest that the distinction between conceptualizing semantic transparency in relatedness versus compositional terms is indeed crucial: In tasks that require access to the whole-word compound semantics (as the eyetracking task investigated here), processing times are predicted by the semantic relatedness between the constituent meanings and whole-word compound meaning (compare Schmidtke et al. (2018)). On the other hand, in tasks that require fast lexical access (as the lexical decision tasks investigated here), processing times are not predicted by semantic relatedness, but by the ease of combining the constituent meanings (Günther \& Marelli, 2018a). Finally, in explicit judgment tasks without specific instructions (as the rating study investigated here), judgments are predicted by by virtually all aspects of compound semantics, including both semantic relatedness and compositionality (compare Marelli and Baroni (2015)). 
We also demonstrated that focusing only on single aspects of semantic transparency, without considering the other possible characterizations, might lead to suboptimal results: In the screening steps of our analyses, there are cases where parameters on their own predict behavioral data, but are not included in the optimal model for the data (for example modifier composition for explicit ratings, or constituent similarity for the double lexical decision task). This again shows the importance of investigating semantic transparency as a multi-dimensional construct.

\section{Open practices statement}

The data and materials for all experiments, as well as the scripts to generate all included measures, are available at https://doi.org/10.6084/m9.figshare.8295101.

\section{Appendix A: Analysis of the GhoSt-NN dataset}

The GhoSt-NN dataset (Schulte im Walde et al., 2016) includes expert ratings on the constituent-wise compositionality of 868 German compounds, on a scale from 1 (definitely semantically opaque) to 6 (definitely semantically transparent). Note that the polarity of this scale is different from the semantic transparency ratings reported in the main article (Böhl, 2007), where higher values indicated semantic opacity. The ratings were collected from 8 to 13 experts (linguists and computational linguists). Since they had the option not to give a judgment, the dataset contains values collected from and averaged over 5 to 13 ratings. The shared set of items between the GhoST-NN dataset and our dataset of 1,810 compounds is very small (58 items). However, by relying on the extended set of 40,475 additional compounds, we are able to derive semantic transparency measures for 690 of the included compounds. It remains that the analyses presented here cannot be considered as a validation study of our core dataset. For all analyses reported here, we applied the same forward model selection technique described in the main article.

Table 9 Results of the likelihood ratio tests testing whether the semantic measures on their own improve the baseline model for modifier ratings

\begin{tabular}{lllll}
\hline parameter & $b$ & $t$ & $X^{2}(1)$ & $p$ \\
\hline modifier relatedness & 2.40 & 16.39 & 226.06 & $<.001$ \\
modifier composition & 1.76 & 8.17 & 61.90 & $<.001$ \\
compound compositionality & 2.25 & 11.95 & 129.53 & $<.001$ \\
constituent similarity & 0.93 & 5.60 & 30.36 & $<.001$
\end{tabular}

Table 10 Parameters for the final model optimally predicting explicit modifier ratings, after model criticism

\begin{tabular}{lllll}
\hline step & parameter & $b$ & $t$ & $p$ \\
\hline & intercept & 3.42 & 14.24 & $<.001$ \\
& head frequency & 0.06 & 3.17 & .002 \\
& compound frequency & -0.10 & -6.15 & $<.001$ \\
1. & modifier relatedness & 1.88 & 10.05 & $<.001$ \\
2. & compound compositionality & 0.74 & 2.75 & .006 \\
3. & head relatedness & -0.29 & -1.69 & .092 \\
\hline
\end{tabular}

Parameters for semantic measures are listed in order of their inclusion in the model. The parameter for head relatedness added in the forward selection is no longer significant after model criticism

\section{Modifier Ratings}

The baseline model for modifier ratings contained parameters for head frequency and compound frequency. In the first screening step, the inclusion of four different parameters significantly improved the baseline model: modifier relatedness, modifier composition, compound compositionality, and constituent similarity (see Table 9).

The final model, after model criticism, contained two semantic effects: modifier relatedness and compound compositionality (see Table 10). We interpret this result as reflecting the rating task administered to the participants: On the one hand, their task was framed in terms of the compositionality of compounds. On the other hand, the actual instruction provided was to give ratings on a scale ranging from "definitely semantically opaque" to "definitely semantically opaque", suggesting judgments of constituent-wise semantic transparency. This underspecification of the task instruction could explain the fact that we observe effects of both compound compositionality and modifier relatedness (note however that the compound compositionality effect does not need to follow from the framing of the task, since we also find this effect for "standard" semantic transparency ratings in our main article). Critically, the participant's task was not to indicate the contribution of the modifier constituent to a compositional compound meaning - in this case,

Table 11 Results of the likelihood ratio tests testing whether the semantic measures on their own improve the baseline model for head ratings

\begin{tabular}{lllll}
\hline parameter & $b$ & $t$ & $X^{2}(1)$ & $p$ \\
\hline head relatedness & 2.90 & 15.49 & 203.47 & $<.001$ \\
head composition & 2.35 & 8.08 & 60.99 & $<.001$ \\
compound compositionality & 1.68 & 6.93 & 46.03 & $<.001$ \\
constituent similarity & 1.23 & 6.06 & 35.67 & $<.001$
\end{tabular}


Table 12 Parameters for the final model optimally predicting explicit head ratings, after model criticism

\begin{tabular}{lllll}
\hline step & parameter & $b$ & $t$ & $p$ \\
\hline & intercept & 4.59 & 10.50 & $<.001$ \\
& compound frequency & -1.25 & -5.98 & $<.001$ \\
& modifier family size & 0.00 & 1.47 & .142 \\
& head family size & -0.00 & -0.76 & .446 \\
1. & head relatedness & 2.35 & 10.50 & $<.001$ \\
2. & compound compositionality & 0.89 & 2.58 & .010 \\
3. & modifier relatedness & -1.00 & -4.34 & $<.001$ \\
\hline
\end{tabular}

Parameters for semantic measures are listed in order of their inclusion in the model

we would have expected to observe an effect of modifier composition on the ratings.

\section{Head Ratings}

The baseline model for modifier ratings contained parameters for compound frequency, as well as modifier family size and head family size. In the first screening step, the inclusion of four different parameters significantly improved the baseline model, mirroring the modifier rating results: head relatedness, head composition, compound compositionality, and constituent similarity (see Table 11).

The final model, after model criticism, contained three semantic effects: head relatedness, modifier relatedness, and compound compositionality (see Table 12). Note that the family size effects are no longer significant in the final model; however, excluding these effects does not affect the significance level of the remaining parameters.

The results for the head ratings mirror the effects for modifier ratings, in that we observe an effect of head relatedness and compound compositionality. Additionally, we observe a negative effect of modifier relatedness (higher values come with lower transparency ratings). In fact, the corresponding head relatedness effect was included also in the modifier rating results before it was eliminated in the model criticism step (see Table 10). This suggests that participants were indeed specific in making transparency judgments for the constituent in question, and gave the highest ratings when only this constituent was semantically transparent.

\section{References}

Akaike, H. (1998). Information theory and an extension of the maximum likelihood principle. In Selected papers of Hirotugu Akaike, (pp. 199-213). New York: Springer.
Baayen, R. H. (2008). Analyzing linguistic data: A practical introduction to statistics using $R$. Cambridge: Cambridge University Press.

Baayen, R. H., \& Milin, P. (2010). Analyzing reaction times. International Journal of Psychological Research, 3(2), 12-28.

Bayen, R. H., Piepenbrock, R., \& Gulikers, L. (1995). The CELEX lexical data base (CD-ROM). University of Pennsylvania: Linguistic Data Consortium.

Balota, D. A., Yap, M. J., Hutchison, K. A., Cortese, M. J., Kessler, B., Loftis, B., \& Treiman, R. (2007). The English Lexicon Project. Behavior Research Methods, 39, 445-459.

Baroni, M., Bernardini, S., Ferraresi, A., \& Zanchetta, E. (2009). The WaCky wide web: a collection of very large linguistically processed web-crawled corpora. Language Resources and Evaluation, 43, 209-226.

Baroni, M., Dinu, G., \& Kruszewski, G. (2014). Don't count, predict! A systematic comparison of context-counting vs. contextpredicting semantic vectors. In Proceedings of ACL 2014, (pp. 238-247). East Stroudsburg: ACL.

Bates, D., Mächler, M., Bolker, B., \& Walker, S. (2015). Fitting linear mixed-effects models using lme4. Journal of Statistical Software, $67(1), 1-48$.

Bauer, L. (2009). Lieber, R., \& Štekauer, P. (Eds.) Typology of compounds, (pp. 343-356). New York: Oxford University Press.

Beauvillain, C. (1996). The integration of morphological and wholeword form information during eye fixations on prefixed and suffixed words. Journal of Memory and Language, 35, 801820.

Böhl, A. (2007). German compounds in language comprehension and production. (Doctoral dissertation, Westfälische WilhelmsUniversität, Münster, Germany. Available online at: https:// nbn-resolving.org/urn:nbn:de:hbz:6-77509571368).

Bronk, M., Zwitserlood, P., \& Bölte, J. (2013). Manipulations of word frequency reveal differences in the processing of morphologically complex and simple words in German. Frontiers in Psychology, 4, 546.

Brysbaert, M., Buchmeier, M., Conrad, M., Jacobs, A. M., Bölte, J., \& Böhl, A. (2011). The word frequency effect. Experimental Psychology, 58, 412-424.

Collins, A. M., \& Loftus, E. F. (1975). A spreading-activation theory of semantic processing. Psychological Review, 82, 207-428.

Dinu, G., Pham, N., \& Baroni, M. (2013). DISSECT: Distributional semantics composition toolkit, (pp. 31-36). East Stroudsburg: ACL.

Dohmes, P., Zwitserlood, P., \& Bölte, J. (2004). The impact of semantic transparency of morphologically complex words on picture naming. Brain and Language, 90, 203-212.

Downing, P. (1977). On the creation and use of English compound nouns. Language, 53, 810-842.

Frisson, S., Niswander-Klement, E., \& Pollatsek, A. (2008). The role of semantic transparency in the processing of English compound words. British Journal of Psychology, 99, 87-107.

Gagné, C. L., \& Shoben, E. J. (1997). Influence of thematic relations on the comprehension of modifier-noun combinations. Journal of Experimental Psychology: Learning, Memory, and Cognition, 23, 71-87.

Gagné, C. L., \& Spalding, T. L. (2009). Constituent integration during the processing of compound words: Does it involve the use of relational structures? Journal of Memory and Language, 60, 20-35.

Gagné, C. L., Spalding, T. L., \& Schmidtke, D. (in press). LADEC: Large database of English compounds. Behavior Research Methods.

Guevara, E. (2010). A regression model of adjective-noun compositionality in distributional semantics. In Proceedings of the 2010 workshop on geometrical models of natural language semantics, pp 33-37. 
Gumnior, H., Bölte, J., \& Zwitserlood, P. (2006). A chatterbox is a box: Morphology in German word production. Language and Cognitive Processes, 21, 920-944.

Günther, F., Dudschig, C., \& Kaup, B. (2015). LSAfun-An R package for computations based on latent semantic analysis. Behavior Research Methods, 47, 930-944.

Günther, F., \& Marelli, M. (2016). Understanding karma police: The perceived plausibility of noun compounds as predicted by distributional models of semantic representation. PLOS ONE, 11(10), e0163200. https://doi.org/10.1371/journal.pone.0163200.

Günther, F., \& Marelli, M. (2018a). Enter sandman: Compound processing and semantic transparency in a compositional perspective. Journal of Experimental Psychology: Learning, Memory, and Cognition, Advance online publication. https://doi.org/10.1037/xlm0000677.

Günther, F., \& Marelli, M. (2018b). The language-invariant aspect of compounding: Predicting compound meanings across languages. In Cabrio, E., Mazzei, A., \& Tamburini, F. (Eds.) Proceedings of the 5th Italian conference on computational linguistics (CLiC-IT 2018), (pp. 230-234). Turin: Accademia University Press.

Günther, F., Rinaldi, L., \& Marelli, M. (2019). Vector-space models of semantic representation from a cognitive perspective: A discussion of common misconceptions. Perspectives on Psychological Science, Advance online publication. https://doi.org/10.1177/1745691619861372.

Günther, F., Smolka, E., \& Marelli, M. (2019). 'Understanding' differs between English and German: Capturing systematic language differences of complex words. Cortex, 116, 168-175.

Henrich, V., \& Hinrichs, E. (2011). Determining immediate constituents of compounds in GermaNet. In Proceedings of the 2011 international conference on recent advances in natural language processing (RANLP 2011), (pp. 420-426).

Hollis, G. (2017). Estimating the average need of semantic knowledge from distributional semantic models. Memory \& Cognition, 45, $1350-1370$.

Hyönä, J., Bertram, R., \& Pollatsek, A. (2005). Identifying compound words in reading: An overview and a model. In Underwood, G. (Ed.) Cognitive processes in eye guidance. Oxford: Oxford University Press.

Jackendoff, R. (2002). Foundations of knowledge. Oxford: Oxford University Press.

Ji, H., Gagné, C. L., \& Spalding, T. L. (2011). Benefits and costs of lexical decomposition and semantic integration during the processing of transparent and opaque English compounds. Journal of Memory and Language, 65, 406-430.

Jones, M. N., Hills, T. T., \& Todd, P. M. (2015). Hidden processes in structural representations: A reply to Abbott, Austerweil, and Griffiths (2015). Psychological Review, 122, 570-574.

Juhasz, B. J. (2007). The influence of semantic transparency on eye movements during English compound word recognition. In Eye movements: A window on mind and brain, (pp. 373-389). Amsterdam: Elsevier.

Juhasz, B. J. (2016). Experience with compound words influences their processing: An eye movement investigation with English compound words. The Quarterly Journal of Experimental Psychology, Advance online publication. https://doi.org/10.1080/17470218. 2016.1253756

Juhasz, B. J., Lai, Y.-H., \& Woodcock, M. L. (2015). A database of 629 English compound words: ratings of familiarity, lexeme meaning dominance, semantic transparency, age of acquisition, imageability, and sensory experience. Behavior Research Methods, 47, 1004-1019.

Kim, S. Y., Yap, M. J., \& Goh, W. D. (2018). The role of semantic transparency in visual word recognition of compound words: A megastudy approach, Behavior Research Methods, Advance online publication. https://doi.org/10.3758/s13428-018-1143-3.
Kintsch, W. (1988). The use of knowledge in discourse processing: A construction-integration model. Psychological Review, 95, 163 182.

Koester, D., Gunter, T. C., \& Wagner, S. (2007). The morphosyntactic decomposition and semantic composition of German compound words investigated by ERPs. Brain and Language, 102, 64-79.

Kuperman, V., Schreuder, R., Bertram, R., \& Baayen, R. H. (2009). Reading polymorphemic Dutch compounds: toward a multiple route model of lexical processing. Journal of Experimental Psychology: Human Perception and Performance, 35, 876895.

Kuznetsova, A., Brockhoff, P. B., \& Christensen, R. H. B. (2017). lmerTest package: Tests in linear mixed effects models. Journal of Statistical Software, 82(13), 1-26.

Landauer, T. K., \& Dumais, S. T. (1997). A solution to Plato's problem: The Latent Semantic Analysis theory of acquisition, induction, and representation of knowledge. Psychological Review, 104, 211-240.

Libben, G. (1998). Semantic transparency in the processing of compounds: Consequences for representation, processing, and impairment. Brain and Language, 61, 30-44.

Libben, G. (2014). The nature of compounds: A psychocentric perspective. Cognitive Neuropsychology, 31, 8-25.

Libben, G., Gibson, M., Yoon, Y. B., \& Sandra, D. (2003). Compound fracture: The role of semantic transparency and morphological headedness. Brain and Language, 84, 50-64.

Lorenz, A., Heide, J., \& Burchert, F. (2014). Compound naming in aphasia: Effects of complexity, part of speech, and semantic transparency. Language, Cognition and Neuroscience, 29, 88-106.

Lorenz, A., \& Zwitserlood, P. (2014). Processing of nominal compounds and gender-marked determiners in aphasia: Evidence from German. Cognitive Neuropsychology, 31, 40-74.

Lynott, D., \& Ramscar, M. J. A. (2001). Can we model conceptual combination using distributional information? In Proceedings of the 12th Irish conference on artificial intelligence and cognitive science, (pp. 1-10). Ireland: Maynooth.

Mandera, P., Keuleers, E., \& Brysbaert, M. (2017). Explaining human performance in psycholinguistic tasks with models of semantic similarity based on prediction and counting: A review and empirical validation. Journal of Memory and Language, 92, $57-78$.

Marelli, M., \& Baroni, M. (2015). Affixation in semantic space: Modeling morpheme meanings with compositional distributional semantics. Psychological Review, 122, 485-515.

Marelli, M., Dinu, G., Zamparelli, R., \& Baroni, M. (2015). Picking buttercups and eating butter cups: Spelling alternations, semantic relatedness, and their consequences for compound processing. Applied Psycholinguistics, 36, 1421-1439.

Marelli, M., Gagné, C. L., \& Spalding, T. L. (2017). Compounding as abstract operation in semantic space: A data-driven, large-scale model for relational effects in the processing of novel compounds. Cognition, 166, 207-224.

Marelli, M., \& Luzzatti, C. (2012). Frequency effects in the processing of Italian nominal compounds: Modulation of headedness and semantic transparency. Journal of Memory and Language, 66, 644-664.

Mikolov, T., Chen, K., Corrado, G., \& Dean, J. (2013). Efficient estimation of word representations in vector space. arXiv: $1301.3781 \mathrm{v} 3$

Monsell, S. (1985). Repetition and the lexicon. In Ellis, A. W. (Ed.) Progress in the psychology of language, (Vol. 1, pp. 147-195). Hove and London: Lawrence Erlbaum Associates Ltd.

Osgood, C. E., \& Hoosain, R. (1974). Salience of the word as a unit in the perception of language. Attention, Perception, \& Psychophysics, 15, 168-192. 
Plag, I. (2003). Word-formation in English. Cambridge: Cambridge University Press.

Pollatsek, A., \& Hyönä, J. (2005). The role of semantic transparency in the processing of Finnish compound words. Language and Cognitive Processes, 20, 261-290.

R Core Team (2017). R: A language and environment for statistical computing. Vienna, Austria. Retrieved from. http://www. R-project.org/.

Rayner, K. (1975). The perceptual span and peripheral cues in reading. Cognitive Psychology, 7, 65-81.

Rayner, K. (1998). Eye movements in reading and information processing: 20 years of research. Psychological Bulletin, 124, 372-422.

Sahel, S., Nottbusch, G., Grimm, A., \& Weingarten, R. (2008). Written production of German compounds: Effects of lexical frequency and semantic transparency. Written Language \& Literacy, 11, 211-227.

Sandra, D. (1990). On the representation and processing of compound words: Automatic access to constituent morphemes does not occur. The Quarterly Journal of Experimental Psychology Section A, 42, 529-567.

Schäfer, M. (2018). The semantic transparency of English compound nouns. Berlin: Language Science Press.

Schmid, H. (1995). Improvements in part-of-speech tagging with an application to German. Proceedings of the ACL SIGDATWorkshop, Dublin, Ireland.

Schmidtke, D., \& Kuperman, V. (2019). A paradox of apparent brainless behavior: The time-course of compound word recognition. Cortex, 116, 250-267.

Schmidtke, D., Van Dyke, J. A., \& Kuperman, V. (2018). Individual variability in the semantic processing of English compound words. Journal of Experimental Psychology: Learning, Memory, and Cognition, 44, 421-439.

Schulte im Walde, S., Hätty, A., Bott, S., \& Khvtisavrishvili, N. (2016). GhoSt-NN: A representative gold standard of German noun-noun compounds. In: Proceedings of the 10th international conference on language resources and evaluation (pp. 2285-2292), Portoroz, Slovenia.

Seidenberg, M. S., \& McClelland, J. L. (1989). A distributed, developmental model of word recognition and naming. Psychological Review, 96, 523-568.

Smolka, E., \& Libben, G. (2017). Can you wash off the hogwash?semantic transparency of first and second constituents in the processing of German compounds. Language, Cognition and Neuroscience, 32, 514-531.

Spalding, T. L., Gagné, C. L., Mullaly, A. C., \& Ji, H. (2010). Relationbased interpretation of noun-noun phrases: A new theoretical approach. Linguistische Berichte Sonderheft, 17, 283-315.

Thagard, P. (1984). Conceptual combination and scientific discovery. In Asquith, P., \& Kitcher, P. (Eds.) PSA: Proceedings of the Biennial meeting of the Philosophy of Science Association, (Vol. 1, pp. 3-12). East Lansing: Philosophy of Science Association.

Turney, P. D., \& Pantel, P. (2010). From frequency to meaning: Vector space models of semantics. Journal of Artificial Intelligence Research, 37, 141-188.

Underwood, G., Petley, K., \& Clews, S. (1990). Searching for information during sentence comprehension. In From eye to mind: Information acquisition in perception, (pp. 191-203). Amsterdam: Elsevier Science.

Westbury, C. (2016). Pay no attention to that man behind the curtain: Explaining semantics without semantics. The Mental Lexicon, 11, 350-374.

Williams, E. (1981). On the notions "lexically related" and "head of a word". Linguistic Inquiry, 12, 245-274.

Zwitserlood, P. (1994). The role of semantic transparency in the processing and representation of Dutch compounds. Language and Cognitive Processes, 9, 341-368.

Publisher's note Springer Nature remains neutral with regard to jurisdictional claims in published maps and institutional affiliations. 